\title{
A Biomechanical Invariant for Gait Perception
}

\author{
James E. Cutting, Dennis R. Proffitt, and Lynn T. Kozlowski \\ Wesleyan University
}

\begin{abstract}
Viewers can determine the gender of a walker from sagittally projected, dynamic displays of point-lights attached to prominent joints. This article explores three interrelated approaches in search of a biomechanical invariant that viewers might use. The first, an index of torso structure, accounts for the data handsomely but seems inappropriate because it is not directly revealed in the dynamic stimuli. The second, a dynamic index of visible torsion in the trunk of a walker, also fits the data well but seems to have a logical problem and a difficulty in accounting for performance in certain conditions of several previous studies. The third has the strengths of the first two indices, and it can account for some other data as well. It is the center of moment and is a "deeper," more general description of the invariant. This center is a point around which all movement occurs. It can be thought of as one specification of the gestalt law of common fate and may be helpful for the study of movement perception in general.
\end{abstract}

We are continually impressed by the fact that one can identify a friend at great distance. Across a broad field, for example, individuals are incredibly small-subtending less than $1^{\circ}$ of visual angle-yet their recognition is easily obtained. Since differences in clothing and hairstyle are obscured at such distances, one suspects that it must be the friend's pattern of gait that provides the critical information. Recently, this suspicion was confirmed (Cutting \& Kozlowski, 1977) with a technique whose antecedents are quite old.

For over a century human gait has been studied by using strips or points of light that glow in the dark. Marey (1895/1972, p. 60), for example, attached white tape to the limbs of a walker dressed in a black body stocking. $\mathrm{He}$ then observed the systematic traces left with stroboscopic photography as the walker moved laterally in front of a camera. Later,

This research was sponsored by small research grants from Wesleyan University to the authors. We thank Catharine D. Barclay and David $M$. Stier for their help.

Requests for reprints should be sent to J. Cutting, Department of Psychology, Wesleyan University, Middletown, Connecticut 06457. other researchers used small incandescent bulbs attached to the joints to gain the same effect (for a review, see Bernstein, 1967, p. $3 \mathrm{ff})$. This technique, called cyclography, yields planar projections of the cycles of movement displayed over time. With this technique and others, human gait became one of the most studied of all complex, cyclical movements. Most of this research, however, focused on how the movement is produced. Following Johansson (1973, 1975, 1976), we have chosen a variant of the cyclographic technique to study how gait is perceived.

Glass-bead retroflectant bicycle tape is wrapped around walkers' joints, and bright lights focused on them as they walk in front of a television camera connected to videorecording apparatus. With contrast on the television monitor turned to maximum and with brightness to near minimum, only patches of light can be seen. If the camera is placed slightly out of focus, these globs become quite circular and remain the only sources of information available to the viewer. Thus, all familiarity cues of clothing and hairstyle are removed from the display. Static versions of a walker with these pointlights are seen in Figure 1. 


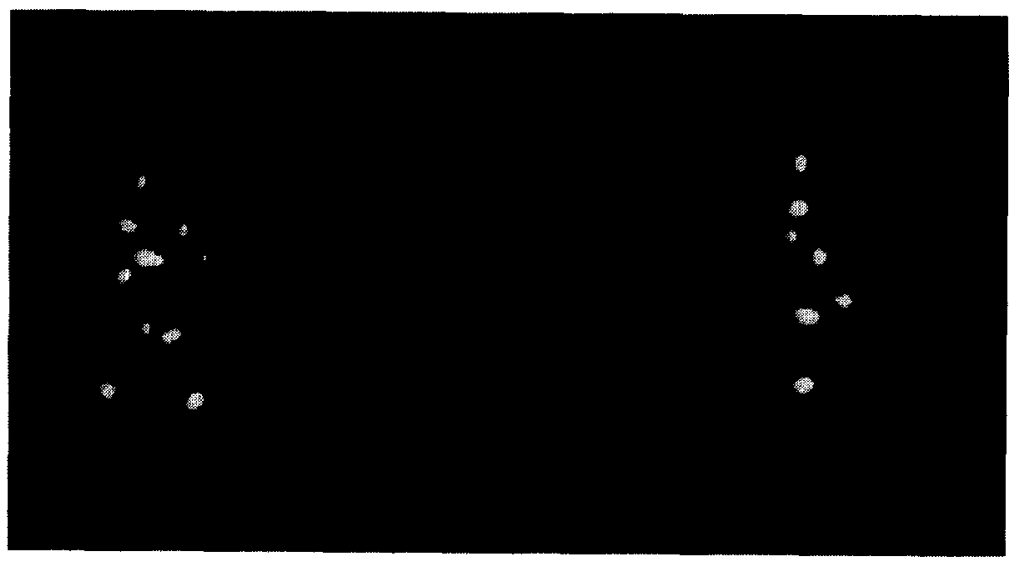

Figure 1. Static approximations of the dynamic point-light display. (The same male walker is presented twice as he walks from left to right, once with arms and legs most outstretched and once with body parts most aligned. Lights occur on the walker's shoulder, elbows, wrists, hip, knees, and ankles. None occurs on the head.)

After demonstrating that viewers could recognize themselves and others from these dynamic point-light displays (Cutting \& Kozlowski, 1977), we began a research program on the identification of a walker's gender (Barclay, Cutting, \& Kozlowski, 1978; Kozlowski \& Cutting, 1977). In a series of 10 experiments we found, among other things, (a) that viewers could indeed identify gender without familiarity cues, (b) that such recognition was possible from dynamic displays but not from static ones like those shown in Figure 1, (c) that no jointlight seemed necessary to the display and that any pair of lights by themselves-such as on the two ankles-seemed sufficient for gender recognition, although the best performances involved lights on both hip and shoulder, and (d) that a series of stimulus degradations interfered with the perceptual process, such as nonnormal variations in walking speed and armswing or limitations of the amount of time viewers could observe the displays. In these studies, however, we were unable to determine what structurally invariant information viewers might be perceiving. After new analyses of the stimuli, we discovered a biomechanical invariant in the gait of male and female walkers that observers can use. What follows is an explication of that invariant. For purposes of exposition, we discuss it in tutorial form, as three different approximations using the same base data. Support for each is given, with new analyses of our previous studies and with evidence from elsewhere.

A caveat. Before beginning the discussion, however, we should state that we are looking for a single aspect of the movement of a human walker that affords gender recognition. Although we acknowledge that viewers may very well be using many sources of information to make their decisions, and may even use different sources on different occasions, we have chosen not to take a particularistic approach. Instead, we are looking for more global information that is distributed throughout the movement. We believe that many of the differences in natural gait may accrue because of underlying structural differences in walkers, not because of particular, epiphenomenal manifestations of movement. The analogy to psycholinguistics is transparent: We are looking for "deep structures" in gait that could yield many "surface structure" forms. Or, put another way, we are looking for differences in "syntactic base" for the production of gait in male and female walkers. Our goal is to provide the most plausible and parsimonious description of the underlying structure. We will be choosing among three similar descriptions of the same source differences. A subsequent description is, we believe, always "deeper" than a previous one. "Deeper" descriptions are preferable on logical grounds 
because they are more general and abstract, but at this point there appears to be no empirical way to separate them from more superficial descriptions. Thus, the brunt of this article should be viewed as an attempt to provide a heuristic means for the perception of form through movement in general, not simply gender recognition for gaits of various kinds.

\section{First Approximation: Shoulder/Hip Ratio}

Barclay et al. (1978) measured the shoulder and hip widths of their 14 walkers. These seven males and seven females were approximately the same height and weight. ${ }^{1}$ Columns 1 and 2 of Table 1 show those measurements. In this sample the shoulders of males averaged $3.6 \mathrm{~cm}$ broader than those of females, $t(12)=5.63, p<.001$, and the hips of females averaged $.7 \mathrm{~cm}$ broader than those of males, $t(12=-.79$, ns. These differences are consistent with many reports of gender differences in bone structure, and the hip measurements, if anything, may underestimate differences normally found between males and females (see Gray's Anatomy, 1901/1977, pp. 178-181). When shoulder width is divided by hip width, shown in column 3 , this ratio can be used as an index of the torso structure for all walkers.

These mean values, 1.10 for males and .99 for females, compare favorably with two sets of measurements of human proportion taken elsewhere. The first comes from the sketches of Albrecht Dürer (1528/1972). Following the lead of Leonardo, Dürer made an extensive study of the relations of human form. In his Dresden sketchbooks are 48 males and 22 females who can be measured in a manner similar to the way we have measured our walkers. The mean proportions (and standard errors of the mean) are $1.19(.01)$ and .97 (.01), respectively. In general, Dürer drew well-muscled males who were taller than his women, thus creating the greater difference across sexes than seen in our sample. The second set of anthropometric data is reported by Faust (1977). She found larger proportions than ours, 1.39 for young adult males and 1.30 for young adult females.
This increase, however, is due to her use of bi-iliac (pelvic width) measurements, which underestimate hip width by about $25 \%$. With this measurement difference in mind, one can see that our indices are quite similar to hers.

We developed another index to assess the identifiability of each walker in these studies. It is shown in column 8 of Table 1. Here, the standard conditions of all experiments of Barclay et al. are combined and identifiability expressed as a function of the percentage of all trials in which each walker was identified as male. Thus, when females were consistently and correctly identified as females, their scores are well below 50\%; males, of course, generally have scores well above $50 \%$. When this index of identifiability, or "percent maleness" score, ${ }^{2}$ is compared with the index of torso structure, a striking correspondence is found $(r=.84), t(12)=$ $5.4, p<.001 .^{3}$ It appears that gender identification of a walker may depend on structural differences between males and females, especially the relation of shoulders to hips.

\section{A Problem}

Barclay et al. (1978), however, noted that if this relation between torso structure and identifiability is not fortuitous, then it is somewhat paradoxical. All walkers in their study were viewed from the side. Thus, differences in widths of shoulders and hips appear to be hidden from the viewer since only the right shoulder and right hip were seen. If structural differences contribute to identifiability of males and females, then

\footnotetext{
1 Note that since males in this country are usually larger than females by $5 \%-10 \%$, this is an unusual sample, and it provided a strong test for the hypothesis that gender recognition was possible from dynamic point-light displays without familiarity cues.

2 Of course, a percent femaleness score could have just as easily been used, yielding negative correlations.

3 It is interesting that when a correlation is calculated for identifiability of each walker against a dummy, two-state variable that indicates the walker's true gender, $r=86$, nearly the same value as the correlation for indices of identifiability and torso structure. Thus, true gender is no better a predictor of performance than is torso structure.
} 
Table 1

Measurements and Indices for the 14 Walkers of Barclay, Cutting, and Kozlowski (1978)

\begin{tabular}{|c|c|c|c|c|c|c|c|c|}
\hline \multirow[b]{2}{*}{ Walker } & \multicolumn{3}{|c|}{ Physical measures (in $\mathrm{cm}$ ) } & \multicolumn{3}{|c|}{ Torsion measures $\left({ }^{\circ}\right)$} & \multirow{2}{*}{$\begin{array}{c}\text { Center of } \\
\text { moment: } \\
\text { Index } 3\end{array}$} & \multirow{2}{*}{$\begin{array}{l}\text { Index of } \\
\text { Identif- } \\
\text { ability }\end{array}$} \\
\hline & Shoulders & Hips & Index 1 & Angle A & Angle B & Index 2 & & \\
\hline \multicolumn{9}{|l|}{ Female } \\
\hline $\begin{array}{l}1 \mathrm{TR} \\
2 \mathrm{IB} \\
3 \mathrm{DD} \\
4 \mathrm{AL} \\
5 \mathrm{JS} \\
6 \mathrm{EL} \\
7 \mathrm{CP}\end{array}$ & $\begin{array}{l}38.0 \\
38.5 \\
38.5 \\
37.0 \\
38.5 \\
37.5 \\
39.5\end{array}$ & $\begin{array}{l}39.5 \\
42.0 \\
35.0 \\
36.0 \\
40.0 \\
39.0 \\
40.5\end{array}$ & $\begin{array}{r}.96 \\
.92 \\
1.10 \\
1.03 \\
.96 \\
.96 \\
.98\end{array}$ & $\begin{array}{l}170.2 \\
162.1 \\
163.1 \\
162.0 \\
162.5 \\
161.0 \\
159.4\end{array}$ & $\begin{array}{l}159.5 \\
151.6 \\
151.8 \\
161.7 \\
153.0 \\
153.5 \\
144.9\end{array}$ & $\begin{array}{r}11.7 \\
10.5 \\
11.3 \\
.3 \\
9.5 \\
7.5 \\
14.5\end{array}$ & $\begin{array}{l}.49 \\
.48 \\
.52 \\
.51 \\
.49 \\
.49 \\
.49\end{array}$ & $\begin{array}{l}34.6 \\
20.0 \\
36.6 \\
45.7 \\
47.4 \\
25.5 \\
35.5\end{array}$ \\
\hline $\begin{array}{l}M \\
S D\end{array}$ & $\begin{array}{r}38.2 \\
.8\end{array}$ & $\begin{array}{r}38.8 \\
2.5\end{array}$ & $\begin{array}{l}.99 \\
.06\end{array}$ & $\begin{array}{r}162.9 \\
3.4\end{array}$ & $\begin{array}{r}153.7 \\
5.5\end{array}$ & $\begin{array}{l}9.3 \\
4.5\end{array}$ & $\begin{array}{l}.49 \\
.01\end{array}$ & $\begin{array}{r}35.0 \\
9.9\end{array}$ \\
\hline \multicolumn{9}{|l|}{ Male } \\
\hline $\begin{aligned} 8 \mathrm{RE} \\
9 \mathrm{RK} \\
10 \mathrm{EA} \\
11 \mathrm{DW} \\
12 \mathrm{RR} \\
13 \mathrm{AM} \\
14 \mathrm{RW}\end{aligned}$ & $\begin{array}{l}40.5 \\
40.0 \\
41.0 \\
43.0 \\
43.0 \\
44.0 \\
41.0\end{array}$ & $\begin{array}{l}38.0 \\
38.0 \\
36.5 \\
38.5 \\
40.0 \\
38.0 \\
38.0\end{array}$ & $\begin{array}{l}1.07 \\
1.05 \\
1.12 \\
1.12 \\
1.08 \\
1.16 \\
1.08\end{array}$ & $\begin{array}{l}177.6 \\
170.3 \\
169.0 \\
174.0 \\
166.6 \\
173.1 \\
177.3\end{array}$ & $\begin{array}{l}156.7 \\
154.5 \\
150.1 \\
159.7 \\
148.3 \\
158.6 \\
155.8\end{array}$ & $\begin{array}{l}20.9 \\
15.8 \\
18.9 \\
14.3 \\
18.3 \\
14.5 \\
21.7\end{array}$ & $\begin{array}{l}.52 \\
.51 \\
.53 \\
.53 \\
.52 \\
.54 \\
.52\end{array}$ & $\begin{array}{l}52.0 \\
54.0 \\
68.9 \\
67.8 \\
73.8 \\
75.5 \\
59.5\end{array}$ \\
\hline $\begin{array}{l}M \\
S D\end{array}$ & $\begin{array}{r}41.8 \\
1.5\end{array}$ & $\begin{array}{r}38.1 \\
1.0\end{array}$ & $\begin{array}{r}1.10 \\
.04\end{array}$ & $\begin{array}{r}172.6 \\
4.2\end{array}$ & $\begin{array}{r}154.8 \\
4.2\end{array}$ & $\begin{array}{r}17.8 \\
3.0\end{array}$ & $\begin{array}{l}.52 \\
.01\end{array}$ & $\begin{array}{r}64.5 \\
9.4\end{array}$ \\
\hline
\end{tabular}

Note. Index 1 is calculated by dividing shoulder width by hip width (see Faust, 1977, pp 66-67). Index 2 is calculated by subtracting Angle B from Angle A (see Figure 3). Index 3 is calculated by dividing shoulder width by the sum of shoulder and hip widths. This is the measure from the point in the spine directly between the shoulders to the center of moment, calculated as a proportion of torso length (see Figure 4 and Footnote 9). The index of identifiability is the percentage of all responses that 102 observers identified each walker as male.

a This walker never swung her arms when she walked.

their specification should allow for views in different planes. Moreover, since gender recognition in these experiments can be accomplished in dynamic but not static arrays, perhaps one should look toward dynamic, and not static, differences between male and female walkers. To do this, one must first understand the step cycle.

\section{The Step Cycle}

Carlsöö (1972), among others (see Inman, 1966; Murray, 1967 ; Napier, 1967 ; Steindler, 1935), provided a useful description of human gait as a modification of the Philippson step cycle (see Grillner, 1975; Sukhanov, 1974). A stride begins when the body sways slightly forward placing the center of gravity in front of the feet. One leg must then swing forward to keep the walker from falling down. At this point, then, the walker's support rests on one leg (thus, it is often called the single support phase). After the swinging foot strikes the ground, both feet remain in contact with the ground (the double support phase) while weight is transferred from the back leg to the front. This pattern is then repeated on the other side, single support phase followed by double support phase. A full step cycle starts from the heel strike of one leg and ends with the next heel strike of that same leg, with all parts of the body returning to their original position. In normal gait the single support phase lasts about twice at long as the double support phase; in running, of course, there is no double support phase.

We suspect that gender recognition of a walker in the dynamic point-light displays is related to the period of the step cycle. 
Walkers in the studies of Kozlowski and Cutting (1977) and Barclay et al. (1978) walked at about 100 paces/minute, slightly above normal (Beckett \& Chang, 1969; Carlsöo, 1972) but well within normal limits (Bornstein \& Bornstein, 1976). At this rate each step cycle takes about $1.2 \mathrm{sec}$ to compete. Gender recognition in these studies took more than $1.6 \mathrm{sec}$ but less than $2.7 \mathrm{sec}$ to accomplish, with a threshold, we argued, somewhere near two step cycles. This amount of time is at least an order of magnitude longer than that Johansson (1976) found for the grosser task of recognizing a human being from the movement of these point-lights.

If gait recognition is dependent on some aspect of the step cycle, it is useful to realize that walking is a synchronous, symmetric pattern of movement. In particular, with the movement of the arms and legs, so goes the movement of the torso.

\section{A Second Approximation: Torso Torque}

The word walk derives from the Old English wealcan, to roll. Indeed, the shoulders and hips roll quite markedly within the step cycle. Consider their movement as shown in the left panel of Figure 2. Schematically represented by straight lines, the hips and shoulders can be seen to move in opposition as the walker steps from one foot to the next ; for example, as the shoulders swing in a clockwise direction, the hips swing counterclockwise, and vice versa. Given this opposition, what can be seen from the side is a constant twisting of the torso, or torque, as the walker steps from foot to foot. This rotation is shown in the top panel of Figure 3; it is about $10^{\circ}$ at both the shoulders and the hips (Murray, 1967). (A small amount of torque can also be measured vertically, as seen in the right panel of Figure 2. This will prove important when we consider the third approximation to the invariant.) Since males have broader shoulders than females, the excursion of the shoulder light, as it oscillates back and forth, should be greater."

A technique was developed to measure the amount of torque visible in the movements of walkers of Barclay et al. (1978). First, the
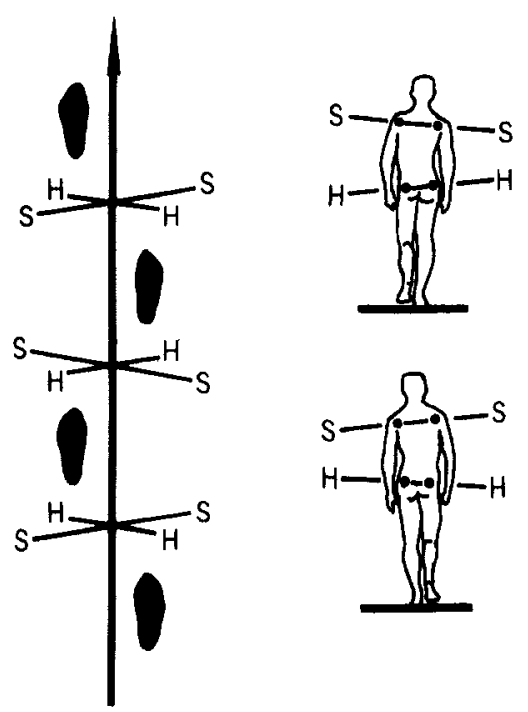

Figure 2. The torso is not held rigid while walking; as the arms and legs work in opposition, the shoulders and hips follow suit. (This opposition can be seen most strikingly viewed from above as a horizontal projection, but it can also be seen from behind as a frontal projection. $\mathrm{S}=$ shoulder; $\mathrm{H}=$ hip. Adapted from Carlsöö, 1972.)

test tape was stopped during playback on the videorecording apparatus so that markings of the shoulder and hip lights could be traced on thin paper placed over the television monitor. Tracings were taken when the walker was judged to have equal distribution of weight on front and back legs during the double support phase. This position accurately describes the limit of torso rotation. Since the position of the forward ankle can be more reliably located than the back ankle, it was chosen as the grounding marker for each tracing. The tape was then manually advanced to take the next tracing.

${ }^{4}$ Of course, given slightly wider hips, females could be said to move their lower torso more than males. After all, the invention of the bustle served to enhance this movement (Flügel, 1930). Nevertheless, since the difference in shoulder widths is more than four times greater than the hip difference in this sample, we concentrate in this section on torque contributed by the shoulders. In passing, however, it is amusing to note that Carlsöö's book is entitled How man moves and indeed may not do justice to women (see Murray, Kory, \& Sepic, 1970). 

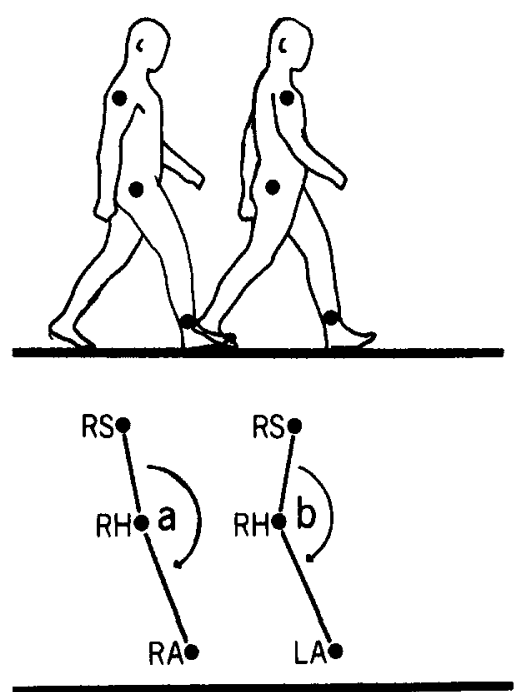

Figure 3. Viewed from the side (as a sagittal projection), torso torque can be measured by subtracting Angle $B$ from Angle $A$. ( $R S=$ right shoulder; $\mathrm{RH}=$ right hip; $\mathrm{RA}=$ right ankle; $\mathrm{LA}=$ left ankle. Adapted from Carlsöö, 1972.)

Thus, right hip, right shoulder, and forward ankle positions were taken for all steps within all trials for every walker. The actual number of steps that could be accurately traced in a single trial varied from four to eight, with a mean of about five.

Next, lines were drawn on the tracing paper, connecting right shoulder to right hip and right hip to forward ankle, thereby making it possible to measure the resulting angle between the three joints for every step. The lower panel of Figure 3 depicts sagittal projections of the angle yielded (a) with the right ankle forward and (b) with the left ankle forward. The former will be called Angle A and the latter Angle B. All Angle A and Angle $B$ measurements were averaged for each walker. The difference between the two provides an index of the degree of torque seen in a full step cycle. Since stride length is constant in normal gait, the torque displayed to the viewer resides entirely within the torso.

Two complete sets of measurements were taken independently by the first author and one assistant. When the difference between Angle A and Angle B was compared for each walker, interobserver reliability was found to be quite high $(r=.84), t(12) 5.6, p<$ .001 . Differences are due primarily to ambiguous hip location for some walkers when their right hand occluded the hip light for some Angle A measurements. Nevertheless, considering the necessarily crude nature of these measurements, agreement between observers is remarkably good. Shown in columns 4 and 5 of Table 1 are the averaged measurements of both observers for Angle A and Angle $B$, and in column 6 is the mean difference in the two angles for each walker. This torque index is correlated with the structure index of shoulder/hip ratios $(r=$ $.47), t(12)=1.85, p<.05$, one-tailed, although the correlation is not strong. Remember, we are assuming that these indices reflect the same structural base.

Torso torque may be the source of structural information critical to the identification of male and female walkers. However, when this torsion index (column 6 of Table 1) and the identifiability index (column 8) are compared for all 14 walkers, a relatively marginal relation between the two measures is found when compared to that with Index 1 $(r=.55), t(12)=2.28, \quad p<.05)$. Close examination of column 6 reveals that the neglible torso rotation of one female walker (Walker 4) markedly weakens the correspondence between the two indices. If she is removed from the analysis, the correlation between torque and identifiability increases considerably $(r=.68), t(11)=3.02, p<$ .01 .

Justification for removing Walker 4 from statistical analysis is twofold. First, it is not clear that she should, in fact, be considered a member of the same population as the other female walkers. Examination of the torsion indices for the seven females shows that she is $2 S D$ below their mean. Second, whereas the armswing of each of the other 13 walkers followed the direction dictated by the shoulder line, she had no armswing at all: Her arms clung straight to her sides throughout all recorded trials, which caused many viewers to laugh when she was on monitor. Her identifiability index, $45 \%$, was not significantly different from chance and suggests that viewers were simply unable to 
classify her. In any case, the extent to which Walker 4's gait deviates from that of the other walkers seems sufficient cause for the exclusion of her data from further statistical consideration.

It appears, then, that torsion may afford gender recognition in these dynamic displays. But before assuming that it is used, we must be sure that no other attribute is used, and then we must seek out corroborating evidence.

\section{Other Attributes}

Walking speed, relative body length, relative stride length, and other variables were also examined. In an earlier study (Kozlowski \& Cutting, 1977) we noted that females walked slightly faster than males. For the 13 walkers remaining in this sample, however, the relation between paces/minute and the identifiability index was negligible $(r=$ $-.16)$. Next, we entertained the idea that relative body lengths might be different for males and females and that this difference might promote recognition. Measurements were taken at the same time as those for hips and shoulders, and the length of the torso made a fraction of the length from shoulder to ankle. When compared with the index of identifiability, the resulting correlation was very small $(r=.05)$. Relative stride length fared little better $(r=.12)$. The failure of the three particularistic variables appears to support our holistic approach.

A fourth factor, however, does relate to identifiability - the visual angle subtended by each walker/stimulus in this sample. Because males and females were recorded in separate sessions and because there were slight differences between recording conditions, males appeared slightly smaller than females (by a mean of $8 \%$ ), an effect opposite to that found in the normal population. Thus, in some sense, this factor is loaded against finding significant results. It is considered again in the next section.

\section{Corroborating Evidence}

A second sample of walkers. The proposition that torso rotation in gait provides
Table 2

Torsion Measurements for the Six Walkers of Kozlowski and Cutting (1977, Experiment 1)

\begin{tabular}{lcccc}
\hline Walker & Angle A & Angle B & $\begin{array}{c}\text { Index 2 } \\
\text { (torsion) }\end{array}$ & $\begin{array}{l}\text { Percent } \\
\text { maleness }\end{array}$ \\
\hline Female & & & & \\
$1 \mathrm{EM}$ & 166.5 & 156.0 & 10.5 & 36 \\
$2 \mathrm{MM}$ & 169.0 & 161.3 & 7.7 & 33 \\
$3 \mathrm{NS}$ & 169.5 & 150.3 & 19.2 & 68 \\
\hline Male & & & & \\
$4 \mathrm{LK}$ & 169.0 & 147.5 & 21.5 & 73 \\
$5 \mathrm{MH}$ & 169.5 & 150.0 & 19.5 & 81 \\
$6 \mathrm{MR}$ & 165.0 & 148.5 & 17.5 & 62 \\
\hline
\end{tabular}

Note. Angles and torsion measurements are in degrees.

a This walker was systematically misidentified as male.

sex-related information is supported by the analysis of the sample of walkers used by Kozlowski and Cutting (1977, Experiment 1). The same torsion index was measured and calculated from tracings taken from the videotape used in that study. The results are shown in Table 2. When this index (column 3 ) is compared with the index of identifiability, or percent maleness (column 4), a remarkable correspondence is seen $(r=.96)$, $t(4)=6.86, p<.001$. Obviously, this effect may be attributable, in part, to the vagaries of a small sample. However, two facts are encouraging. First, Walker 3 of this sample, who was systematically misidentified as male, had a torsion index similar to that of the three males in the sample. Second, there were no differences in visual angle for male and female walker/stimuli in this sample, yet as Barclay et al. (1978) noted, results were quite comparable for both samples.

Since the 6 walkers in this study and the 13 walkers previously discussed (minus the anomalous female with no torsion) were all Wesleyan University undergraduates and since the viewers in all studies also belonged to the same population, combination of the two samples seemed warranted. Taken together, the correlation of the two indices, torso rotation and identifiability, is quite strong $(r=.76), t(17)=4.83, p<.001$. 
Table 3

Torsion Measurements Taken from the Photographs of Muybridge (1901/1955)

\begin{tabular}{lccc}
\hline \multicolumn{1}{c}{ Photograph } & Angle A & Angle B & $\begin{array}{c}\text { Index 2 } \\
\text { (torsion) }\end{array}$ \\
\hline Female & & & \\
$\quad$ Plate 96 $(6,11)$ & 159 & 155 & 4 \\
Plate 98 $(12,6)$ & 168 & 163 & 5 \\
Male & & & \\
Plate 1 $(1,6)$ & 168 & 155 & 13 \\
Plate 2 $(8,1)$ & 176 & 162 & 14 \\
Plate 4 $(8 / 9,2)$ & 172 & 161 & 11 \\
\hline
\end{tabular}

Note. Numbers in parentheses indicate frames used for calculating Angle A and Angle B, respectively. Where more than one number is given, the mean angle for two of Muybridge's frames is given. Measurements vary according to the exact locus determined by the observer. Nevertheless, if isomorphic locales for ankles, hip, and shoulders are taken, mean angle difference remains quite constant.

a This walker is designated by Muybridge as being stout.

b Measurements for this walker were taken just before the double support phase was initiated. No double support frames are shown by Muybridge. Thus, the visible torque shown here may underestimate actual torque.

Nineteenth century photographs. Unexpected support for the torsion index comes from analysis of photographs published by Muybridge (1901/1955). This volume shows nudes engaged in a large variety of physical activities, such as walking, broadjumping, falling down, and picking up laundry. When walkers from Muybridge's volume are measured according to the method described previously, angles and torsion indices are found to be similar to those of our contemporary samples. Since Muybridge often presents no illustrations of the double support phase, few exact comparisons can be made. Nevertheless, shown in Table 3 are measurements of five individuals for whom these measures can be calculated. The generally smaller degree of torque displayed by these walkers may reflect self-consciousness of subjects being photographed nude in Victorian times. Most important, however, is the fact that the mean visible torsion for males exceeds that for females, $t(3)=6.33$, $p<.01$. The applicability of this measure- ment technique to Muybridge's plates provides happy validation for the torsion index; it suggests that its domain is not restricted to a particular sample, or necessarily to our culture and time. ${ }^{\bar{s}}$

Torsion and disruptions of normal gait. Kozlowski and Cutting (1977, Experiment 3 ) had four walkers vary armswing in order to observe its effect on gender recognition. In general, all nonnormal armswings impeded viewer performance; for example, when the four individuals in this sample walked with their hands in their front pockets, with their hands held at their side, or with very exaggerated armswing, viewers could not reliably determine their gender. When armswing was slightly greater than normal, gender recognition was possible $(59 \%)$, but it remained below that for walks with normal armswing $(65 \%)$. If torsion provides the primary cue to viewers as to the sex of a particular walker, perhaps the difference in torsion between males and females is diminished for nonnormal armswing conditions.

To test for this possibility, we took tracings and measurements for all trials used in the test sequence. At the top of Table 4, for each of the five conditions, the average torsion is shown for male and female walkers. ${ }^{6}$ Each of these indices was obtained by subtracting a mean Angle B from a mean Angle $A$, as shown in Figure 3. When the difference in indices for the males and the females (column 3 ) is compared with the overall performance level of all viewers in this study, another striking correlation is seen $(r=$ $.90), t(3)=3.58, p<.05$. Again, the sample size of conditions is very small, and this result should not be overemphasized. Never-

\footnotetext{
5 As suggested in the next section, there remains some possibility that the increased torsion in the males of the Muybridge sample is due to increased walking speed and stride length. Without knowing the exact intervals between frames in his zoopraxographic displays, we can only assume that they were approximately the same for males and females.

6 These measurements are somewhat smaller than those in Table 1. This may be due largely to the fact that they were taken by a different observer. Most important, however, are not the angles themselves but the relations between them.
} 
Table 4

Indices and Results for Kozlowski and Cutting (1977, Experiments 3 and 4)

\begin{tabular}{|c|c|c|c|c|}
\hline \multirow[b]{2}{*}{ Condition } & \multicolumn{2}{|c|}{ Torsion index $\left({ }^{\circ}\right)$} & \multirow{2}{*}{$\begin{array}{l}\text { Difference between } \\
\text { indices }\left({ }^{\circ}\right)\end{array}$} & \multirow{2}{*}{$\begin{array}{l}\text { Overall performance } \\
\text { level of viewers }(\%)\end{array}$} \\
\hline & Female & Male & & \\
\hline \multicolumn{5}{|l|}{ Variation in armswing } \\
\hline $\begin{array}{l}\text { Hands in pockets } \\
\text { Hands at sides } \\
\text { Normal armswing } \\
\text { Large armswing } \\
\text { Exaggerated armswing }\end{array}$ & $\begin{array}{l}4.6 \\
3.0 \\
3.9 \\
8.1 \\
6.2\end{array}$ & $\begin{array}{r}3.8 \\
4.6 \\
9.4 \\
10.4 \\
8.0\end{array}$ & $\begin{array}{r}-.8 \\
1.6 \\
5.5 \\
2.3 \\
1.8\end{array}$ & $\begin{array}{l}46 \\
54 \\
65 \\
59 \\
55\end{array}$ \\
\hline \multicolumn{5}{|c|}{ Variation in walking speed (mean paces/min) } \\
\hline $\begin{array}{r}87 \\
95 \\
103 \\
111 \\
119\end{array}$ & $\begin{array}{l}3.5 \\
5.2 \\
3.9 \\
5.7 \\
5.8\end{array}$ & $\begin{array}{l}3.4 \\
7.1 \\
9.4 \\
9.5 \\
6.7\end{array}$ & $\begin{array}{r}-.1 \\
1.9 \\
5.5 \\
3.8 \\
.9\end{array}$ & $\begin{array}{l}56 \\
57 \\
67 \\
61 \\
68\end{array}$ \\
\hline
\end{tabular}

theless, it may be that deviations in armswing from that for normal gait diminish the usual difference in visible torsion for males and females, and that decrease is associated with a similar decrease in identifiability.

In addition to perturbing armswing, Kozlowski and Cutting (1977, Experiment 4) disrupted normal gait by having walkers walk at different speeds. In general, fasterand slower-than-normal gaits yielded poor gender recognition. Sinilar measures were taken for these stimuli as for the other studies, and those results are shown at the bottom of Table 4. Again, when the difference in torsion indices for males and females is compared with overall performance level, a positive correlation is found $(r=.45)$. Although this correspondence is not statistically reliable, $t(3)=.88$, it is in the predicted direction.

Problems. Support appears strong for torsion as an index on which perceptual judgments about a walker's gender can be made. Nevertheless, there are some problems. First, Kozlowski and Cutting (1977, Experiment 5) demonstrated that accurate gender recognition can be made on the basis of arm movement alone, leg movement alone, or even ankle movement alone. In the last example, when the ankles are the only joints represented in a point-light display, viewers remain significantly above chance in their gender judgments (but see Kozlowski \& Cutting, 1978). It appears difficult to determine the torque of the shoulders against the hips as portrayed by only the movement below the knee.

The second problem is conceptual in nature. The torsion index tells us that males appear to twist their bodies in natural gait more than females, but it does not indicate where that torsion occurs. Considering Figure 3, decreases in Angle B could be attributable to large excursions of the right hip backward or to large excursions of the right shoulder forward. We have argued that since males have broader shoulders than females and since their differences in hip width is very small, it is the shoulder swing that contributes most to this index. Logically, however, one might find a female with wide hips and narrow shoulders who yields an index comparable with that of males. Thus, the torsion index appears vulnerable to differences in locus of torque in a way that we would like it not to be.

What is needed, then, is another approximation of an invariant, one that retains all the positive qualities of visible torque but has neither of these problems. More important, however, we would like to devise a descriptive index applicable to moving objects other than human walkers, objects with neither shoulders nor hips. 
A Third Approximation: Center of Moment

Following Duncker (1939), Johansson (1974; Maas \& Johansson, 1971) described experiments with the point-light technique involving a wheel. One light was placed at the perimeter of the wheel as it was rolled across a flat surface. Viewed from the side, the light described a series of trochoids. Without any information other than this one point, viewer experience was not of seeing a wheel but rather a series of scallops. As soon as another light was placed on the wheel, however, wheellike movement could be seen. Most important to our argument is the fact that this second light need not be at the wheel's hub (Proffitt, Cutting, \& Stier, Note 1). Many other locales on the perimeter, and many places between the hub and the perimeter, will do. The two lights, because of their systematic and periodic movement, describe arcs around the wheel's center. These arcs, taken together, provide invariant information about the object's center of rotation. Borrowing a concept from mechanics, we call this the center of moment. ${ }^{7}$ All objects that move in contact with the ground-and probably all objects that move-have at least one such center, regardless of whether they are rigid or elastic.

The movement of the human body is several orders of magnitude more complex than that of a wheel, but the same principles of structural mechanics apply. Human gait is made up of the rigid motions of four compound pendula (the arms and legs) hinged at the corners of a flat spring (the torso). When all these parts or subsystems are set in motion, the movement is periodic and symmetric about the center of moment of the body as a whole. Thus, specifying the position of the right ankle within the step cycle, for example, automatically and with considerable accuracy specifies the positions of all other body parts. Symmetry, of course, occurs about a plane, axis, or point. It is not surprising to find one locus of symmetry that is midsagittal, following the plane of biological symmetry. There are, however, two other planes of symmetry for gait. A second plane divides the upper torso from the lower. Its approximate locale can be esti- mated from the torsion of a walker, seen in the right panel of Figure 2. The third plane of symmetry divides the walker front from back and can be estimated from Figure 3 . The point of intersection for these three planes is the center of moment. For a walker, all parts of the body can be said to move about it. Perhaps it is a proper specification of the structural invariant we have been looking for.

\section{Centers of Moment and Gravity}

Every moving body, however, has at least two centers, one about which all movement occurs (the center of moment) and another about which all mass is distributed (the center of gravity). For very simple moving bodies these two points are often identical. Consider first a wheel. If the wheel has uniformly distributed mass, then the center of gravity and the center of moment coincide; that is, all movement occurs about that point which is both center of moment and center of mass. Suppose, however, that a weight were attached to the perimeter. The wheel's center of gravity would now lie at some distance away from its previous locale, but the center of moment would remain the same. Consider next a pendulum. The center of gravity of a pendulum, like the asymmetric wheel, is different from its center of moment. The latter lies at the pivot, whereas the former oscillates beneath.

Since the arms and the legs are pendula, their centers of moment are their pivots, the shoulders and hips. Since these four points are also the corners of a flat spring undergoing torsion, their motion (and the motion of the arms and legs) can be further

7 A moment, in engineering mechanics and elastics, is the tendency, or measure of the tendency, to produce motion. The center of moment has often been called the center of movement (Marey, 1895/ 1972, pp. 289-290) in biomechanics, and it can be found in rough form in the sketches of Leonardo and Dürer. It is the center for an arc or system of arcs; for example, a fulcrum is the center of moment of a lever arm, as is an axle for a wheel. It should be noted that centers of moment can, themselves, move in other than simple translatory fashion. 
MALE

FEMALE
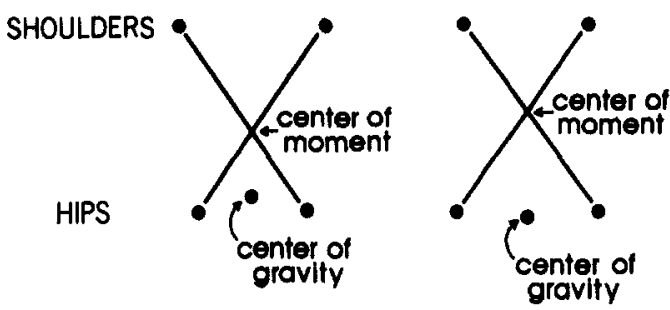

Figure 4. Schematic representation of the torsos of males and females from this sample. (Males have slightly wider shoulders than hips, whereas this relation is reversed for females. Differences are slightly exaggerated here for purposes of clarity. Center of moment is marked as the intersection of stress lines across the torso, acting like a flat spring. Approximate center of gravity is also indicated. Albrecht Dürer, 1528/1972, used a similar technique in locating a center of torsion [pp. 11, 29, 109, and 171] froms widths of shoulders and hips [pp. 139, 141, and 171].)

described as having a higher order center of moment. Its locale can be approximated by drawing stress lines across the diagonals of the torso, as shown in Figure $4 .{ }^{8}$ The intersection is the center of moment, and it is the same point as the intersection of the three planes of symmetry discussed previously. Drawing these as straight lines assumes that the torso, in the shape of an isosceles trapezoid, has uniform elasticity along its length. Although strictly speaking this is not true, there are probably no systematic differences across males and females other than torso structure to weaken this formulation. Given that the shoulders of males are slightly wider than the hips and that the relation is reversed for females, the center of moment will be slightly lower for males than for females by several centimeters.

Shown in the same figure are the approximate locations of the center of gravity for men and women. For both it lies roughly between the hips, but because the thighs are generally less massive for males (Bernstein, 1967 , p. 13), their center of gravity is slightly higher than females'. Thus, the direction of difference for the two centers is opposite across sexes.

The center of gravity plays an important role in gait. It is thrust forward of the sup- port line of the feet in order to initiate and maintain forward momentum. Thus, as Napier (1967, p. 56) suggested, "human walking is a unique activity during which the body, step by step, teeters on the edge of catastrophe," oscillating between falling down and righting itself. The center of gravity, however, may be a much more labile point than the center of moment, as suggested earlier by their structural relations in an asymmetric wheel and in a pendulum. The center of gravity can, for example, lie outside the physical limits of the human body. Thompson (1917/1969, p. 28) noted that although a high jumper throws his body over the bar, he actually throws his center of gravity under it. It would seem, however, that the center of moment for a high jumper would not be wholly different from that for a walker and that, unlike the center of gravity, it may have to go over the bar with him.

\section{Estimation of the Center of Moment}

Given the relations shown in Figure 4, it is possible to locate the center of moment from knowledge of the widths of the shoulders and hips. If shoulder width is represented by $s$ and hip width by $h$, the center of moment, $C_{m}$, can be estimated by Formula 1 as a proportion of the torso length measured down from the shoulder line ${ }^{2}$ :

$$
C_{\mathrm{m}}=s /(s+h)
$$

8 Figure 4 shows the torso as represented in the frontal plane, whereas all viewers in these experiments viewed sagittal projections. In terms of the location of the center of moment, however, this difference is irrelevant: Movement occurs about the center regardless of what plane it is viewed in. Figure 4, then, shows frontal projections for convenience only.

9 The formula is derived as follows: Consider a line dropped from the midpoint between the shoulders to the midpoint between the hips. As it intersects one of the diagonals, it forms two right triangles with parallel bases (one is half of the shoulder width and the other is half of the hip width). If $t$ stands for the length of the torso, and $t C_{\mathrm{m}}$ for the length of the torso from the shoulder line to the center of moment, then the following proportion can be established:

$$
.5 s / t C_{\mathrm{m}}=.5 h /\left(t-t C_{\mathrm{m}}\right) .
$$


The value of $C_{\mathrm{m}}$ for each walker is shown in column 7 of Table 1 . Because this index is so strongly related to the first index $(r=.98)$, when it is compared with the index of identifiability (column 8), a striking correspondence is seen $(r=.86), t(12)=5.84, p<$ .001 . It should be remembered that this index is preferable because it is based on dynamic principles whereas the first index makes no reference to movement. Because the same data are used to compute them, they cannot be separated on any grounds except logical ones.

\section{$C_{\mathrm{m}}$ and Other Factors}

The center of moment is an attractive notion as a possible invariant for determining the gender of a walker from a dynamic pointlight display. It accounts for more of the results in the gait experiments here and elsewhere with reasonable ease, and it has interesting implications for acuity and for movement perception in general. Consider first the problems that the other indices could not handle.

Previous problems. First, it now makes a certain amount of sense that viewers can

\begin{abstract}
Solving this equation for $C_{m}$ yields Formula 1 . However, a better estimate for $C_{m}$ can be made by assuming that the torso is not a flat spring but a pillar with an ellipsoidal cross section of varying dimensions (see Ugural \& Fenster, 1975, p. 180). The essential difference in these two formulations is that the pillar varies not only in width but also in depth. If we assume that the width, or major axis, of the pillar (torso) changes in a linear fashion from shoulder to hip-allowing for no marked indentation at the waist-and that the depth, or minor axis, of the pillar (the front to back measurement of the torso) is proportional to the width, Formula 1 is modified:
\end{abstract}

$$
C_{\mathrm{m}}=s^{k} /\left(s^{k}+h^{k}\right) \text {. }
$$

If the minor axis of the pillar (torso) is assumed to be $50 \%$ of the major axis at all points along its length, $k=1.4$. When Index 3 , calculated in this new manner, is compared with the index of identifiability, the correspondence is essentially the same as before $(r=.85)$. Mean values of this new index for males and females are .53 and .49 , respectively, yielding a small increase in difference between sexes from the original calculation. Given that little is gained by this more complex formulation, for simplicity's sake, Formula 1 appears to suffice. classify walkers by ankle movement alone. The two ankles move about the common center like all other body parts. The fact that gender from ankles is somewhat less determinable than from other body parts may stem from several reasons. One is that ankle movement is a function of several intervening variables (hip and knee movements) as well. Thus, the common mathematical relation of the ankles to the center of moment may be more difficult for the perceiver to determine than that of the knees or of the elbows to the same center. Another reason for the decreased performance in the anklesalone condition may be that since fewer lights were presented to the viewer, less information was available for judgments to be based on. The notion here would be that all joints provide some information about the $C_{\mathrm{m}}$ and that the more lights that are present, the more redundancy there is in the display. Analysis of the data of Kozlowski and Cutting (1977, Table 3) reveals that performance was highly correlated with the number of lights presented (hence, joints represented; $r=.85), t(5)=3.66, p<.01$, yet performance in all conditions was above chance. In other words, we believe that the human skeletal system is constrained enough during natural gait (the most economical form of human locomotion; Beckett \& Chang, 1969; Inman, 1966) that all points of the body orbit about the center of moment. If true, invariant information concerning the center of moment is necessarily distributed throughout all movement, even as far as to the ankles.

A second problem with the torsion index (Index 2) is that Walker 4 (see Table 1) had to be dropped from analysis. With the center of moment as an index, she needed not be eliminated despite her aberrant gait and minimal torsion: Her $C_{\mathrm{m}}$ index was very much between the modes for males and females in this sample, and her identifiability index was in accordance with it.

A third problem with the torsion index is that it seems not to specify the locus of torsion or the differential amounts of twisting at the shoulders or hips. The center of moment, however, is precisely the locale around 
which all torsion occurs. Thus, a female walker with narrow shoulders and broad hips could logically have the same torsion index as a male with broad shoulders and narrow hips, but their centers of moment would differ considerably.

Other results. The center of moment is a structural invariant, and the pendular and torsional movements in walking are aspects of transformational invariants (Pittenger \& Shaw, 1975). With this difference in mind, it is possible to account for many of our previous results.

Logically speaking, since the center of moment is based on shoulder, hip, and torso length measurements and since these do not change in an individual except over long periods of time, the center of moment should remain the same regardless of the activity. More concretely, the $C_{\mathrm{m}}$ should be the same regardless of whether the individual is walking, running, crawling, or riding a bicycle. We do not claim, however, that males and females would be equally identifiable in all types of movement when viewed as a system of point-lights. All these movements relate to the transformational invariants involved. We have chosen to study natural gait because it is the most economical form of human locomotion. Because of this minimal energy principle, we believe that the transformational invariants of natural gait may best reveal the walker's center of moment. When these invariants are tampered with, perception is likely to change. Consider four cases in which it does. The first two are temporal and the last two are spatial alterations of the transformational information.

The temporal tamperings are those of varying duration and rate of presentation of the point-light displays. Barclay et al. (1978, Experiment 1) found that at least $2 \mathrm{sec}$ of dynamic display time are needed before a viewer can accurately judge gender. This commutes to, roughly, two step cycles needed for recognition and suggests that the difference in centers of moment for males and females is quite small (a point to which we return below) and must be carefully calibrated across a sizable slice of time. A second temporal deformation is that of slowing the display rate, as was done by Barclay et al. (1978, Experiment 2). This also reduces performance to near chance. In both cases temporal aspects of the transformational invariants were varied, and the result appears to be that of making the precise locale of the structural invariant, $C_{\mathrm{m}}$, more difficult to abstract.

The spatial tamperings are those of varying amplitude of certain pendular movements and separational integrity of point-lights. Kozlowski and Cutting (1977, Experiment 3 ) had their walkers vary armswing during gait. It appears that the greater the deviation from the norm, the worse gender recognition was. We argue that each walker's $C_{\mathrm{m}}$ remains constant across these conditions but that the disruption of natural armswing (an aspect of the transformational invariant in gait) disrupts perception. Barclay et al. (1978, Experiment 3) found that another degradation, blurring the relation between the various point-lights, also impeded recognition. We argue that the enlarged globs obscure the natural pendular and torsional movements in gait, even though the center of moment of such a diffuse system is likely to be the same as for its discrete-light counterpart.

In summary, then, the center of moment is a structural invariant that does not guarantee accurate gait perception. Given that walking is a dynamic event, the transformational invariants must be considered as well. In the present article, however, we have been focusing only on the structural invariant information.

Measured differences in gait of other samples. Murray and her colleagues (Murray, 1967 ; Murray, Drought, \& Kory, 1964; Murray et al., 1970) have made extensive measurements of male and female walkers of various heights and ages. Although she found lateral hip and shoulder rotations to be comparable for both sexes, the amount of upper body sway was $65 \%$ greater for males. This sway is the amount that the head and neck move from side to side over the midline during the gait cycle. She concluded:

The marked decreased lateral motion of the head of women, as compared to men, appears to be a 
feminine attitude of locomotion. In keeping with the demands for stability, the mass center of the body must shift from side to side in order that the line of gravity of the body fall within the eccentrically placed bases of support. However, which part of the body shifts laterally appears to be an optional and attitudinal characteristic of gait. It appears to be a masculine attitude to show greater lateral shifting of the head and thorax, and less lateral shifting of the pelvis. Conversely, the feminine attitude appears to be characterized by less lateral shifting of the head and thorax, but greater lateral shifting of the pelvis. (Murray et al., 1970, p. 647)

Since the head and neck are attached to the spine at the level of the shoulders and since the relations among all three are relatively fixed during gait, this lateral motion in the upper body must be occurring about the center of moment. Since the $C_{\mathrm{m}}$ is relatively farther away from the shoulders in males than in females, there is a longer moment arm to generate this sway. Hence the sway is greater. Since the $C_{\mathrm{m}}$ is nearer to the hips in males than in females, the relation between movements is reversed, with women generating more sway than men. Both sways are occurring primarily in the frontal plane, as shown schematically for a male walker in the right panel of Figure 2. Since our walkers were viewed in the sagittal plane, this movement would be revealed in a vertical shortening and lengthening of the torso. The shoulders of males would thus move up and down more than those of females, and the hips of females more than of males. Thus, we suggest that the lateral sway Murray measured is not as optional and attitudinal as she stated. It appears to be a consequence of locational differences in center of moment.

The fact that rotational twist is the same at the shoulders and hips for both males and females is not embarrassing to our formulation. Murray measured only the angle of torsion, not the excursion of the shoulder and hip surfaces. Given equal torsion but unequal widths, male shoulders may move laterally more than females, and female hips may move laterally slightly more than males. ${ }^{10}$

Acuity and gender differences. The fact that the center of moment can be expressed as a fraction of torso length (column 7 of Table 1), suggests that the proximities of the walker to the camera during recording and of the viewer to the monitor during testing matter not all all. Indeed, the results of Kozlowski and Cutting (1977, Experiment 5) support this latter point; there, because viewers sat in a large lecture hall at greatly different distances from monitors, visual angle varied from about $3^{\circ}$ to one third of a degree. It is surprising that those sitting in the back row were nearly as good at judging sex of walkers as those in the front row (61\% vs, $65 \%)$. What makes this back-row feat even more remarkable is that the difference in centers for males and females is no more than $2 \%$ of the height of the dynamic stimulus. If a viewer were sitting quite far from the monitor and the entire stimulus subtended a visual angle of only about one-half degree, then the difference in locale for centers of moment subtends only about $30 \mathrm{sec}$ of an arc. This might appear to render implausible the notion that center of moment affords gender recognition since Haber and Hershenson (1973, p. 113) gave this value as the limit of acuity. However, we are making no statement that the center of moment is seen in isolation. Instead, we are saying that the whole of the dynamic point-light display is seen moving about it. Acuity is thus summoned only to the extent that differences in movements can be seen, not with respect to differences in where the movement is generated.

It should be remembered that just as listeners need no conscious knowledge of syntax and its relation to meaning, viewers need no conscious knowledge of $C_{\mathrm{m}}$ and its relation to gender. In fact, none of the 464 viewers of Kozlowski and Cutting (1977) and Barclay et al. (1978) expressed such knowledge, and yet over all trials in which point-light displays were presented without further degradations, the viewers' performance hovered between $65 \%$ and $70 \%$.

${ }_{10}$ Another factor separates Murray's result from our own. Her participants walked at rates of 112 to 118 paces/minute. As Table 4 shows, these rates appear to diminish torque differences in our sample, as they may have in hers. Thus, the equivalent torques that she found may be artifacts of her choice of walking speed. 
Common fate. One of the lesser developed gestalt laws is the law of common fate, or uniform destiny. Wertheimer (1923/ 1939) seems to have spoken of it so loosely that, as Johansson (1950) noted, Koffka (1935) did not even bother to list it in his Principles of Gestalt Psychology. Nonetheless, we believe this notion to be integrally related to the center of moment. All pointlights in the dynamic displays can be said to have a common fate in that the fate, or movement, of every light at every point in time is a function of their common center of moment. ${ }^{11}$ The weakness in the notion of common fate is that it failed to specify, in a noncircular fashion, what it is that all coherently moving points might share. The $C_{\mathrm{m}}$ is one such specification for lights moving in relation to one another. Note, however, that a center of moment does not specify the type of movement that all points share, merely that all points within the system have some mathematically constant relation with the single point.

\section{Summary}

Individuals viewing a videotape display of a dynamic point-light sequence can recognize the distinctive patterns of movement belonging to male and female walkers. This article gives three interrelated approximations in search of a biomechanical invariant that afforded this aspect of gait recognition. The first considered structural differences between the torsos of men and women. Although this index was strongly correlated with viewer performance, as information that makes no reference to movement we argue that it was inappropriate to the dynamic stimulus. The second approximation, that of visible torque in walkers' torsos, accounted for most of the results. It was somewhat embarrassed, however, by the fact that information from outside the torso yielded accurate judgments of walkers' gender. As well as providing an account for these results, the third approximation accounted for the others. The center of moment is a dynamically based invariant grounded in the law of common fate. It accounts for nearly $75 \%$ of all variance in the data, without overt appeal to pos- sible sociocultural differences in the gaits of males and females. Physically, it is not represented by a light, but all lights move about it in a coherent and symmetric fashion. The complex mathematical relations needed to measure its exact locale are beyond the scope of this article, and thus the $C_{m}$ discussed here remains an approximation and a theoretical construct. Nevertheless, for a walker, the locus of the center of moment can be estimated (a) from knowledge of the widths of shoulders and hips and (b) from knowledge that the torso acts like a flat spring with limbs moving symmetrically about it. This third approximation encompasses the first two; it accounts for all data that they account for and more, and it provides a heuristic means for studying how motion perception might be accomplished. Thus, the $C_{\mathrm{m}}$ is a "deeper" description than the others. We expect that it is fully applicable to the movement of most nonhumanlike objects. Proffitt et al. (Note 1) provide one such analysis for the movement of wheellike objects.

11 A converging operation on the veracity of this view is that Cutting (1978, in press) has, through the use of exaggerated differences in centers of moment, synthesized on a computer hypernormal male and female walkers represented as a system of lights orbiting about their centers in pendular and ellipsoidal fashions.

\section{Reference Note}

1. Proffitt, D. R., Cutting, J. E., \& Stier, D. M. The perception of zeheel-generated motions. Manuscript submitted for publication, 1978.

\section{References}

Barclay, C. D., Cutting, J. E., \& Kozlowski, L. T. Temporal and spatial factors in gait perception that influence gender recognition. Perception $\mathcal{E}$ Psychophysics, 1978, 23, 145-152.

Beckett, R., \& Chang, K. An evaluation of the kinematics of gait by minimum energy. In $D$. Bootzin \& H. C. Muffley (Eds.), Biomechanics. New York: Plenum Press, 1969.

Bernstein, N. The co-ordination and regulation of movements. Oxford: Pergamon Press, 1967.

Bornstein, M. H., \& Bornstein, H. G. The pace of life. Nature, 1976, 259, 557-559.

Carlsöö, S. How man moves. London: Heinemann, 1972. 
Cutting, J. E. Generation of synthetic male and female walkers through manipulation of a biomechanical invariant. Perception, in press.

Cutting, J. E. A program to generate synthetic walkers as dynamic point-light displays. Behavior Research Methods \& Instrumentation, 1978, 10 91-94.

Cutting, J. E., \& Kozlowski, L. T. Recognizing friends by their walk: Gait perception without familiarity cues. Bulletin of the Psychonomic Society, 1977, 9, 353-356.

Duncker, K. Induced motion. In W. D. Ellis (Ed.), A sourcebook of Gestalt psychology. London: Routledge \& Kegan Paul, 1939.

Dürer, A. The human figure: the Dresden sketchbooks. New York: Dover, 1972. (Originally published 1528.)

Faust, M. S. Somatic development of adolescent girls. Monographs of the Society for Research in Child Development, 1977, 42, Serial No. 169.

Flügel, J. C. The psychology of clothes. London: Hogarth Press, 1930.

Gray, H. Anatomy, descriptive and surgical (15th ed.). New York: Bounty, 1977 (Originally published, 1901.)

Grillner, S. Locomotion in vertebrates. Physiological Reviews, 1975, 55, 247-304.

Haber, R. N., \& Hershenson, M. The psychology of visual perception. New York: Holt, Rinehart \& Winston, 1973.

Inman, V. T. Human locomotion. Canadian Medical Association Journal, 1966, 94, 1047-1054.

Johansson, G. Configuration in event perception. Uppsala, Sweden: Almqvist \& Wiksell, 1950.

Johansson, G. Visual perception of biological motion and a model for its analysis. Perception $\&$ Psychophysics, 1973, 14, 210-211.

Johansson, G. Vector analysis in visual perception of rolling motion. Psychologische Forschung, $1974,36,311-319$.

Johansson, G. Visual motion perception. Scientific American, 1975, 232(6), 76-89.

Johansson, G. Spatio-temporal differentiation and integration in visual motion perception. Psychological Research, 1976, 38, 379-393.

Koffka, K. Principles of Gestalt psychology. New York: Harcourt, 1935.

Kozlowski, L. T., \& Cutting, J. E. Recognizing the sex of a walker from a dynamic point-light display. Perception \& Psychophysics, 1977, 21, $575-580$.

Kozlowski, L. T., \& Cutting, J. E. Recognizing the gender of walkers from point-lights mounted on ankles: Some second thoughts. Perception \&' Psychophysics, 1978, 5, 459.

Maas, J. B., \& Johansson, G. Motion perception I: 2-dimensional motion perception. Boston: Houghton Mifflin, 1971. (Film)

Marey, E. J. Movement. New York: Arno Press \& New York Times, 1972. (Originally published in 1895).

Murray, M. P. Gait as a total pattern of movement. American Journal of Physical Medicine, 1967, 46, 290-333.

Murray, M. P., Drought, A. B., \& Kory, R. C. Walking patterns of normal men. Journal of Bone and Joint Surgery, 1964, 46-A, 335-360.

Murray, M. P., Kory, R. C., \& Sepic, S. B. Walking patterns of normal women. Archives of Physical Medicine and Rehabilitation, 1970, 51, 637650.

Muybridge, E. The human figure in motion. New York: Dover, 1955. (Originally published, 1901.)

Napier, J. The antiquity of human walking. Scientific American, 1967, 216(4), 56-66.

Pittenger, J. B., \& Shaw, R. E. Aging faces as viscal-elastic events: Implications for a theory on nonrigid shape perception. Journal of Experimental Psychology: Human Perception and Performance, 1975, 1, 374-382.

Steindler, A. Mechanisms of normal and pathological locomotion in man. Springfield, Ill.: Charles C Thomas, 1935.

Sukhanov, V. B. General system of symmetrical locomotion of terrestrial vertebrates and some features of movement of lower tetrapods. New Delhi, India: Amerind, 1974.

Thompson, D. W. On growth and form. London: Cambridge University Press, 1969. (Originally published, 1917.)

Ugural, A. E., \& Fenster, S. K. Advanced strength and applied elasticity. New York: American Elsevier, 1975.

Wertheimer, M. Laws of organization in perceptual form. In W. D. Ellis (Ed.), A source book of Gestalt psychology. London: Routledge \& Kegan Paul, 1939. (Originally published in German, 1923.)

Received October 20, 1977 